\title{
Veronika Černušková, Judith L. Kovacs und Jana Plátová, gemeinsam mit Vít Hušek, Hgg.: Clement's Biblical Exegesis: Proceedings of the Second Colloquium on Clement of Alexandria (Olomouc, May 29-31, 2014), Supplements to Vigiliae Christianae 139, Leiden (Brill) 2017, 399 S., ISBN 978-90-04-33123-5, € 147,--.
}

Besprochen von Dietmar Wyrwa: Humboldt-Universität zu Berlin,Berlin, Germany, E-Mail:wyrwa@bbaw.de

https://doi.org/10.1515/zac-2018-0019

Seit einigen Jahren macht eine Gruppe jüngerer tschechischer Forscherinnen und Forscher durch bemerkenswerte Aktivitäten auf dem Gebiet der Clemens-Studien auf sich aufmerksam. Zu diesen Aktivitäten gehören nicht nur Monographien, Tagungsbeiträge oder sonstige Publikationen sowie Seminare und spezielle Veranstaltungen, sondern sogar, was von außen als ein besonderes verlegerisches Wagnis erscheint, eine mit Einführungen und Anmerkungen versehene tschechische Übersetzung der Werke des Clemens von Alexandrien nebst griechischem Text. Der hier anzuzeigende Band geht auf eine solche internationale Tagung zurück, die von Angehörigen dieses Forschungskreises in erweiterter Besetzung unter dem Dach der Czech Patristic Society und dem Centre of Patristic, Medieval and Renaissance Texts der Palacký University Olomouc in Olomouc (Olmütz) am 29.-31. Mai 2014 durchgeführt wurde. Es war die zweite, ausschließlich Clemens gewidmete Tagung, und es spricht für das Ansehen, das die tschechische Gruppe in der internationalen Fachwelt genießt, dass wiederum fast die gesamte Elite der Clemens-Spezialisten in Olomouc versammelt war; eine Liste der Teilnehmenden ist im Vorwort abgedruckt. Während die erste Begegnung, das im Oktober 2010 ebendort veranstaltete Colloquium Clementinum 1, sich speziell auf das Siebente Buch der Stromateis des Clemens konzentrierte, haben die Verantwortlichen des Colloquium Clementinum 2, Veronika Černušková (Olomouc, CZ), Judith L. Kovacs (Charlottesville, USA) und Jana Plátová (Olomouc, CZ), für die Folgeveranstaltung ein breiteres Thema gewählt: Clemens' Gebrauch und Interpretation der christlichen Bibel. Diesem Gegenstand ist, wie die Veranstalterinnen zu Recht meinen, in der Clemens-Forschung weniger Beachtung zuteil geworden als etwa seinem Verhältnis zu paganen, zumal philosophischen Quellen, obwohl es nicht weniger von grundlegender Bedeutung ist. Mit der Veröffentlichung der Kongressbeiträge möchten sie das Verständnis von Clemens' Schriftgebrauch fördern und hoffen, einen Anstoß zu weiteren Untersuchungen auf diesem Gebiet zu geben.

Das Konzept zur Durchführung der Tagung wie auch zur anschließenden Publikation lehnt sich eng an das bewährte Muster des ersten Colloquium Clementinum an. Zur Einführung dient eine Sichtung des Bestandes von Clemens' Bibelauslegung samt Forschungsüberblick von Kovacs sowie eine umfassende 
Bibliographie zum speziellen Thema von Plátová. Die anschließenden Vorträge sind in drei Blöcken präsentiert: Der erste Teil, „Clement’s Exegetical Methods“, behandelt methodologische Zusammenhänge von Clemens’ Bibelauslegung in religionsgeschichtlichen, begriffsgeschichtlichen und theologiegeschichtlichen Vergleichen, der zweite Teil, „Clement between Philosophy and Biblical Theology“, geht den Wechselwirkungen zwischen philosophischem Material und Bibelverständnis in Clemens’ Denken nach, der dritte Teil, „Clement's Exegesis of Particular Biblical Texts“, widmet sich konkreten Auslegungen einzelner biblischer Bücher, bestimmter Perikopen oder spezieller Bibelverse. Drei Indices schließen den Band ab.

Es ist aus Raumgründen nicht möglich, alle 14 Beiträge detailliert zu referieren, doch soll versucht werden, ein klares Bild von dem hier dokumentierten Forschungsstand zu geben und wenigstens einige Beobachtungen zu nennen. Im Vorwort des Bandes geben die Herausgeberinnen selbst ein kurzes Resümee aller Vorträge. Eine Gemeinsamkeit, die bei allen Referenten anzutreffen ist und sich auch in dem wertvollen Forschungsbericht von Kovacs immer mehr für die neuere Zeit abzeichnet, ist der völlige Verzicht auf Verdammungsurteile über Clemens' exegetisches Bibelverständnis. Dass Clemens auf dem Wege der allegorischen Methode eine doppelte Sinnebene der Bibel angenommen hat, eine vordergründige und eine höhere, wird nicht mehr, wie es zumal bei protestantischen Theologen lange selbstverständlich war, als verderblicher, toxischer Infekt angesehen, sondern in seinem spirituellen Potential gewürdigt. Besonders die Beiträge des ersten Teils zeugen davon. Alain Le Boulluec (Paris) behandelt Clemens’ Konzept der „symbolischen Form“ (,genre symbolique“), indem er, was bisher nicht so klar gesehen wurde, die Verbindungen mit der komparativen Mythologie eines Cornutus oder eines Plutarch aufweist, in denen Clemens' Ausführungen zu verorten sind. Verschlüsselnde Redeweisen oder zeichenhafte Objekte und Handlungen seien nicht nur im biblischen Bereich, sondern auch, wie Clemens anerkennend referiert, in der paganen Welt anzutreffen, aber Clemens übersteigert den Rahmen der komparativen Mythologie dadurch, dass er diesen Tendenzen das höhere Alter der biblischen Tradition entgegenstellt und die verhüllenden Mitteilungen der Bibel auf den Sohn Gottes als den Urheber zurückführt. Ilaria L.E. Ramelli (Mailand) untersucht das einschlägige Wortmaterial, das Clemens

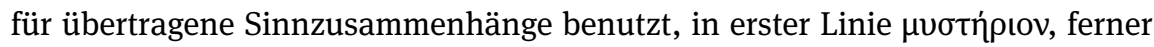

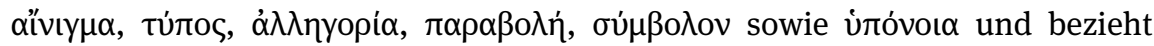
dabei philosophische und kulturgeschichtliche Traditionen mit ein, zuletzt rekurriert sie noch auf Clemens' nur schattenhaft greifbaren Lehrmeister Pantänus. Auch sie betont die spirituelle Leistung dieses Verfahrens, das in der Lage ist, den Leser zur höchsten Erkenntnis zu führen. Marco Rizzi (Mailand) möchte Clemens' eigentümliche Stellung innerhalb der gemeinsamen alexandrinischen 
Auslegungstradition zwischen Philo und Origenes näher bestimmen. Philo richte seine Bibelexegese, Rizzi zufolge, sowohl nach innen an eine jüdische Hörerschaft wie nach außen an pagane Leser der umgebenden griechischen Kultur, um auf dem ethischen Weg der Gesetzesobservanz die Seele zum Aufstieg zu Gott zu leiten, während für Origenes die Bibel nur innerhalb der christlichen Kirche ihren Platz habe, weil nur mit den Augen des Glaubens der geistliche Gehalt der Schrift, der nichts anderes ist als die Offenbarung Christi, ergriffen werden und im spirituellen Fortschritt wachsen kann. Clemens hingegen, so Rizzi, sei eine universale Sicht zu eigen, die auf die gesamte Menschheit blickt, indem er einen berufenen Lehrer als Seelenführer ins Spiel bringt, der in Entsprechung zur heilsgeschichtlichen Pädagogik des Logos die Bibel in aufeinander folgenden Stufen mit Hilfe philosophischer Mittel als Wegweisung zur Erkenntnis der Wirklichkeit im Ganzen im Logos-Christus erschließt.

Den zweiten Teil des Tagungsbandes bestreiten wiederum drei Vorträge. Johannes A. Steenbuch (Copenhagen) beobachtet, dass für Clemens die vom Logos inspirierte Bibel und die „Stimme des Herrn“ (,voice of the Lord“) nicht einfach identisch sind, sondern in dialektischer Beziehung unterschieden werden müssen, und er sieht diesen Gebrauch durch die apophatische Theologie sowie durch den Symbolcharakter der Sprache begründet. Dass die verhüllende religiöse Sprache nicht nur, was Clemens ja oft sagt, den Zweck hat, die Unwürdigen abzuhalten und die Befähigten anzuspornen, sondern ihre Bedeutung viel grundsätzlicher anzusetzen ist, ist daraus zu ersehen. Diese wichtigen Beobachtungen könnten indessen noch durch Auswertung beiläufiger sprachphilosophischer Bemerkungen (etwa Stromateis 8,23,1 und Stromateis 6,126,4, aber nicht nur

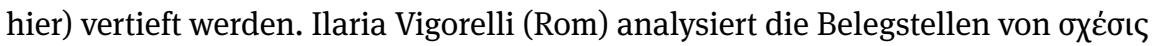
in Clemens' Euvre, die dort theologisch besonders relevant sind, wo sie die wechselseitigen Relationen von Gott-Vater und Sohn Gottes in der einen Gottheit beschreiben. Wirkliches Neuland betritt Matyáš Havrda (Prag) mit seiner Untersuchung über das sog. Achte Buch der Stromateis. Havrda, der sich schon zuvor in Aufsätzen zum Problem dieses Überlieferungskomplexes geäußert hat und von dem jüngst eine bahnbrechende Monographie zum Gegenstand erschienen ist, ${ }^{1}$ wertet Stromateis 8 als einen Auszug aus einem Handbuch zur Theorie des Beweises, dem wahrscheinlich Galens verlorenes philosophisches Hauptwerk De demonstratione zugrunde lag. Es ist geradezu spannend $\mathrm{zu}$ verfolgen, wie er minutiös am Text zeigt, dass das erste Kapitel Clemens' christliche Intention verrät, die Beweistheorie für seine exegetischen Zwecke dienstbar $\mathrm{zu}$ machen,

1 Matyáš Havrda, The So-Called Eighth Stromateus by Clement of Alexandria: Early Christian Reception of Greek Scientific Methodology (Philosophia Antiqua 144; Leiden, 2016). 
und dass auch im weiteren Verlauf, obwohl die Argumente nirgends direkt christlich adaptiert sind, einige winzige Zwischenbemerkungen oder Glossen christlichen Einschlag aufweisen.

Der dritte und letzte Teil des Bandes enthält sechs Beiträge zu speziellen Auslegungen bestimmter Bibeltexte. Annewies van den Hoek (Cambridge, USA) baut eine Notiz von André Méhat ${ }^{2}$ zur Häufigkeit der Zitate aus Proverbia in Hinblick auf Clemens' Zitationstechnik in allen ihren relevanten Aspekten im großen Stil aus (Verweis auf Méhat, S. 182 [Anm. 5]), wobei sie Tabellen und Schautafeln in fünf Appendizes folgen lässt. Der eigentümliche Sachverhalt ist der, dass die Häufigkeit der Zitate kontinuierlich über die Bücher Protrepticus, Paedagogus und Stromateis 1 und 2 ansteigt, um nach der höchsten Dichte in Stromateis 2 drastisch in Stromateis 3 abzufallen und danach fast ganz zu verebben. Méhat hatte mit diesem Argument die Chronologie der Entstehung der Werke abgesichert; Van den Hoek fragt darüber hinaus nach den Gründen, warum Clemens' Benutzung des Proverbien-Buches so plötzlich abbricht, und zieht als Möglichkeiten in Betracht, dass er bei der Abfassung der späteren Bücher kein Bedürfnis mehr verspürte, den Reichtum dieser Schrift auszuschöpfen, oder dass er keinen Zugang mehr zu einer gut ausgestatteten Bibliothek hatte. Zu Letzterem noch eine Bemerkung: Dem Rezensenten scheint es ganz unwahrscheinlich, dass Clemens das Proverbien-Buch, selbst als er Alexandrien verlassen hatte, nicht mehr zugänglich gewesen sein sollte, auch wenn dieser Gesichtspunkt in anderen, nicht kompatiblen Zusammenhängen schon geltend gemacht worden ist - seine nächste Station war immerhin, soweit wir wissen, Jerusalem, wo Alexander eine Bibliothek aufgebaut hatte.

Veronika Černušková (Olomouc) untersucht Clemens’ Auslegung der Bergpredigt. Während einzelne Verse zu den am häufigsten herangezogenen oder angespielten Stellen in Clemens' Werk überhaupt gehören, gibt es andere Verse der Bergpredigt, die er gar nicht erwähnt. Die Rekurse lassen sich Černušková zufolge um drei Themenkreise gruppieren: Unterdrückung unstatthafter Begierden, Streben nach Gemeinschaft mit Gott und Erfüllung von Gottes Gebot der Feindesliebe. Und auch sie unterbreitet das Material mit ausführlichen Tabellen. Piotr Ashwin-Siejkowski (London) geht Clemens' Rezeption des Johannes-Evangeliums nach, das, wie er feststellt, nicht unter den von ihm am häufigsten herangezogenen Schriften rangiert (an erster Stelle stehen die Paulus-Briefe und unter den Evangelien das Matthäus-Evangelium; Johannes-Evangelium, Johannes-Briefe und Johannes-Apokalypse gehen Clemens' Ansicht nach auf ein und denselben

2 André Méhat, Étude sur les „Stromates“ de Clément d’Alexandrie (Patristica Sorbonensia 7; Paris, 1966), 52-54. 
Verfasser zurück). Insgesamt mache Clemens nur einen unsystematischen und beiläufigen Gebrauch vom Johannes-Evangelium; die größte Bedeutung komme dem Prolog zu, der jedoch nicht in erster Linie das Fundament für die Logos-Lehre sei (dafür kämen Philo und der Mittelplatonismus in Betracht), aber den christlichen Offenbarungsgedanken mit dem heilsgeschichtlichen Aufriss und der Inkarnation als Höhepunkt beisteuere. Ebenfalls mit dem Prolog beschäftigt sich Miklós Gyurkovics (Nyíregyháza, Ungarn). Er macht darauf aufmerksam, dass sich Clemens in der Auslegung von Joh 1,1.3b-4a.18 philosophische Konzepte zu „place“ zunutze macht, um auf den drei Ebenen der göttlichen, der intelligiblen und der materiellen Wirklichkeit konsistente Strukturen von Raum und Existenz freizulegen, die im göttlichen Logos verankert sind.

Davide Dainese (Bologna) konzentriert sich auf das literarische Problem der sog. Adumbrationes, indem er Clemens' Bemerkungen $\mathrm{zu}$ den dort aufgeführten Stellen des 1. Johannes-Briefes (besonders Adumbrationes 3,2,7; 3,5,8-14) mit anderen Passagen in Clemens' Euvre, wo dieselben Verse behandelt werden, vergleicht. Er kommt zu dem Ergebnis, dass die Adumbrationes nicht, wie bisher angenommen, ein in lateinischer Übersetzung und Bearbeitung überliefertes Stück aus den verlorenen Hypotyposen, sondern exegetische Vorarbeiten zu Problemlösungen seien, die Clemens mehrmals zum Thema De principiis angekündigt hatte. Ob er solche systematischen Erörterungen in einem eigenständigen, heute verlorenen Werk unter dem Titel De principiis ausgeführt hat oder ob sie Eingang in die Stromateis gefunden haben, will Dainese für zukünftige Forschungen offen halten. Dem Rezensenten muten freilich nicht selten Daineses Textauswertungen willkürlich und spekulativ an; überzeugend wirkt seine Dekonstruktion der traditionellen Zuweisung nicht. Das kann aus Raumgründen hier nicht im einzelnen ausgeführt werden und müsste angesichts der Tatsache, dass Dainese mehrmals auf seine jüngst erschienene Publikation, einer italienischen Übersetzung mit ausführlicher Einleitung zu den Adumbrationes ${ }^{3}$, verweist, auf eine breitere Basis gestellt werden.

Wie Judith L. Kovacs den Band eröffnet hat, so beschließt sie ihn auch mit einer konzisen Skizze zur Wertschätzung des Apostels Paulus, „des göttlich inspirierten Apostels“ („,Divinely Inspired“ Paul“), den Clemens mit großem Abstand häufiger als irgendeinen anderen Autor herbeizieht. Kovacs möchte erhellen, wie stark sich Clemens' Paulusrezeption im Dialog bzw. in Auseinandersetzung mit „heterodoxen“ Christen, d.h. mit Valentinianern, mit Antinomisten sowie radikalen Asketen, andererseits mit schlichten Gläubigen und mit griechischen Philo-

3 Davide Dainese, Hg., Clemente Alessandrino: Adombrazioni (Letture cristiane del primo millennio 51; Mailand, 2014). 
sophen bewegt. Seine Lieblingszitate, 1 Kor 1,24, Hebr 1,1, 1 Kor 2,9 und Eph 4,13, markieren eindrücklich die paulinisch untermauerten Schwerpunkte seiner theologische Gesamtsicht: Die Verkündigung Christi als Gottes Kraft und Weisheit ist der heilsgeschichtlich vielfältig in Bibel und Philosophie vorbereitete universale Ruf an alle Menschen, sich einem christlichen Leben zuzuwenden, das in einem fortschreitenden Prozess zu immer größerer Vollkommenheit und zu immer tieferer Gotteserkenntnis führt

Abschließend ist den Herausgeberinnen für die mit Gewinn zu lesende Publikation der Tagungsbeiträge zu danken und der Gruppe der tschechischen Forscherinnen und Forscher insgesamt zu wünschen, dass ihr Engagement in clementinischen Dingen auch weiterhin reiche Früchte zeitigen möge. 